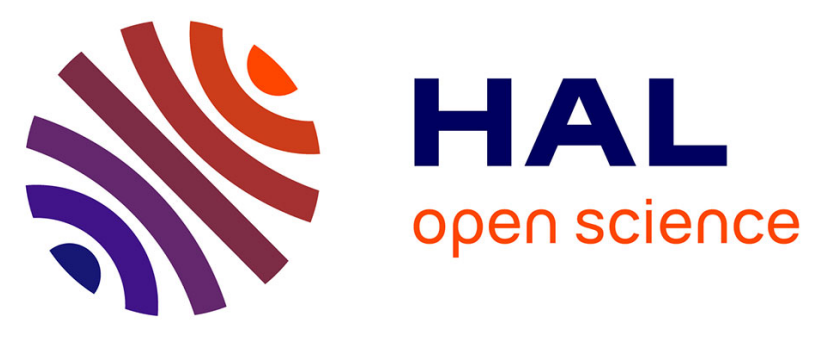

\title{
Untangling structural factors driving genome stabilization in nascent Brassica napus allopolyploids
}

Julie Ferreira de Carvalho, Solenn Stoeckel, Frédérique Eber, Maryse

Lodé-Taburel, Marie-Madeleine Gilet, Gwenn Trotoux, Jérôme Morice, Cyril

Falentin, Anne-Marie Chèvre, Mathieu Rousseau-Gueutin

\section{To cite this version:}

Julie Ferreira de Carvalho, Solenn Stoeckel, Frédérique Eber, Maryse Lodé-Taburel, Marie-Madeleine Gilet, et al.. Untangling structural factors driving genome stabilization in nascent Brassica napus allopolyploids. New Phytologist, 2021, 230 (5), pp.2072-2084. 10.1111/nph.17308 . hal-03227311

\section{HAL Id: hal-03227311 \\ https://hal.science/hal-03227311}

Submitted on 19 May 2021

HAL is a multi-disciplinary open access archive for the deposit and dissemination of scientific research documents, whether they are published or not. The documents may come from teaching and research institutions in France or abroad, or from public or private research centers.
L'archive ouverte pluridisciplinaire HAL, est destinée au dépôt et à la diffusion de documents scientifiques de niveau recherche, publiés ou non, émanant des établissements d'enseignement et de recherche français ou étrangers, des laboratoires publics ou privés. 
DR JULIE FERREIRA DE CARVALHO (Orcid ID : 0000-0001-6200-3344)

Article type : Regular Manuscript

\section{Untangling structural factors driving genome stabilization in nascent Brassica napus allopolyploids}

Julie Ferreira de Carvalho ${ }^{1}$, Solenn Stoeckel ${ }^{1}$, Frédérique Eber ${ }^{1}$, Maryse Lodé-Taburel ${ }^{1}$, Marie-Madeleine Gilet $^{1}$, Gwenn Trotoux ${ }^{1}$, Jérôme Morice ${ }^{1}$, Cyril Falentin ${ }^{1}$, Anne-Marie Chèvre ${ }^{1}$, Mathieu RousseauGueutin $^{1}$

\section{Author affiliation:}

${ }^{1}$ IGEPP, INRAE, Institut Agro, Univ Rennes, 35650 Le Rheu, France

Dr Solenn Stoeckel ORCID : 0000-0001-6064-5941

Dr Anne-Marie Chèvre ORCID: 0000-0001-5312-032X

Cyril Falentin ORCID : 0000-0002-9901-3412

\section{Corresponding authors:}

Dr. Julie Ferreira de Carvalho

Address: IGEPP, INRAE, Institut Agro, Univ Rennes, Le Rheu, France

Phone : +33(0)2 23235997

E-mail : julie.ferreira-de-carvalho@inrae.fr

ORCID: 0000-0001-6200-3344

Dr. Mathieu Rousseau-Gueutin

Address: IGEPP, INRAE, Institut Agro, Univ Rennes, Le Rheu, France

Phone: +33(0)2 23235131

E-mail: mathieu.rousseau-gueutin@inrae.fr

ORCID: 0000-0002-1130-1090

This article has been accepted for publication and undergone full peer review but has not been through the copyediting, typesetting, pagination and proofreading process, which may lead to differences between this version and the Version of Record. Please cite this article as doi: 10.1111/NPH.17308 
Received: 7 January 2021

Accepted: 22 February 2021

\section{SUMMARY}

(1) Allopolyploids have globally higher fitness than their diploid progenitors however, by comparison, most resynthesized allopolyploids have poor fertility and highly unstable genome. Elucidating the evolutionary processes promoting genome stabilization and fertility is thus essential to comprehend allopolyploid success.

(2) Using the Brassica model, we mimicked the speciation process of a nascent allopolyploid species by resynthesizing allotetraploid $B$. napus and systematically selecting for euploid individuals over eight generations in four independent allopolyploidization events with contrasted genetic backgrounds, cytoplasmic donors and polyploid formation type. We evaluated the evolution of meiotic behavior, fertility and identified rearrangements in S1 to S9 lineages, to explore the positive consequences of euploid selection on $B$. napus genome stability.

(3) Recurrent selection of euploid plants for eight generations drastically reduced the percentage of aneuploid progenies as early as the fourth generation, concomitantly with a decrease in number of newly fixed homoeologous rearrangements. The consequences of homoeologous rearrangements on meiotic behavior and seed number strongly depended on the genetic background and cytoplasm donor.

(4) The combined use of both self-fertilisation and recurrent euploid selection, allowed identification of genomic regions associated with fertility and meiotic behavior, providing complementary evidence to explain $B$. napus speciation success.

Keywords: Brassica napus (oilseed rape), euploid selection, fertility, genome stability, homoeologous exchanges, meiotic behavior, polyploidy. 


\section{INTRODUCTION}

All seed plants have experienced at least one episode of Whole-Genome Duplication (WGD) during their evolutionary history (Jiao et al., 2011; One Thousand Plant Transcriptome Initiative, 2019). This process tends to increase genetic and phenotypic diversity at various levels, and has been associated with greater fitness and more diverse ecological niches in polyploids compared to their diploid relatives (Selmecki et al., 2015; Baniaga et al., 2019). These observations are based on the successful outcomes of millions of years of evolution, however immediately after WGD, polyploids have to overcome several challenges due to bearing more than two sets of each chromosome. These sets of chromosomes can be more or less divergent depending on the occurrence of intra or interspecific hybridization before WGD leading to creation of auto or allopolyploids, respectively. To increase its chance of speciation, complex genome stabilization processes must occur rapidly after the formation of an allopolyploid species. Primarily, strict bivalent formation is required for the formation of balanced gametes. However, in the case of allopolyploidy, redundant chromosomes coming from related parental species (homoeologous chromosomes) may pair with each other, and impact meiotic behaviour and plant fertility. Interestingly, the predominance of these events differs greatly among allopolyploids. Generally, multivalent associations are prevented during meiosis in most of natural allopolyploids (Grandont et al., 2013). To explain this mechanism, different hypotheses were proposed: either pre-existing genomic divergence between constitutive diploid progenitors impedes homoeologous pairing, or the presence of a genetic control in progenitors lead to a regulating mechanism of homoeolog pairing in the polyploid context (Mason \& Wendel, 2020). For instance, genetic factors were shown involved in complete or partial homoeologous pairing control respectively in natural wheat allopolyploids and oilseed rape (for review Jenczewski \& Alix, 2004; Griffith et al., 2006; Gonzalo et al., 2019). Contrastingly, in resynthesized allopolyploids (such as Brassica and Tragopogon), illegitimate pairing between homoeologous chromosomes is observed as soon as the first meiosis (Tate et al., 2006; Szadkowski et al., 2010). After numerous recombination events between homoeologs, their global genomic similarity tends to increase, leading to even more illegitimate COs as formulated by Gaeta \& Pires (2010) under the term 'polyploid ratchet'. As expected, because of the presence of univalents and multivalents in various germ cells, viable balanced gametes and fertility are extremely low in resynthesized allopolyploids exhibiting mispairing behaviour. Yet, the early structural dynamics linked to rearrangements between homoeologous genomes and their impact on meiotic instability and fertility stay unexplored in newly formed allopolyploids. Thus, deciphering the evolutionary processes that generated genome stabilization in natural allopolyploids and how this could be achieved in resynthesized ones is essential to fully comprehend early polyploid speciation processes. 
Brassica napus L. (AACC, $2 n=4 x=38$ ) is an allotetraploid resulting from the interspecific hybridization that took place ca. 7500 years ago (Chalhoub et al., 2014) between two closely related diploid species B. rapa (AA, $2 n=2 x=20)$ and $B$. oleracea $(C C, 2 n=2 x=18)$. Spontaneous populations of $B$. napus were so far never uncovered. Recent studies have nonetheless identified the European origin of both $A$ and $C$ subgenomes of B. napus (An et al. 2019; Song et al., 2020). Although homoeolog pairing is limited in natural B. napus, different studies have repeatedly demonstrated the presence of numerous homoeologous translocations, limited in size but contributing to intraspecific diversity in B. napus (Chalhoub et al., 2014; Samans et al., 2017; Higgins et al., 2018; Song et al., 2020). Resynthesized crosses between the diploid Brassica species have been created to mimic the first steps of allopolyploid speciation to inform on the role of homoeologous rearrangements in meiosis control, genome stabilization and seed production. Homoeologous rearrangements and high levels of aneuploids have been observed in different resynthesized lines of B. napus (Song et al., 1995; Gaeta et al., 2007; Szadkowski et al., 2010; Xiong et al., 2011; Rousseau-Gueutin et al., 2017). Homoeologous rearrangements promote drastic genome instability as $50 \%$ of gametes may present homoeologous exchanges as soon as the first meiosis (Szadkowski et al., 2010). Depending on progenitors and type of gamete formation used in resynthesized crosses, homoeologous rearrangements amplify in a non-random fashion in the first generations (Szadkowski et al., 2011; Rousseau-Gueutin et al., 2017) and alter meiosis and seed production (Szadkowski et al., 2010, 2011; Girke et al., 2012; Jesske et al., 2013; Rousseau-Gueutin et al., 2017). Thus, it is paramount to investigate how the presence of non-reciprocal homoeologous exchanges impact meiosis and fertility: either by the size and position of these rearrangements along the genome, disturbing homoeologous pairing; or by modifying allele dosage and genetic mechanisms controlling meiosis and homoeologous pairing. Additionally, in the first generations, a significant proportion of aneuploid individuals were described causing supplementary instability (Xiong et al., 2011). These aneuploids result from chromosome mispairing and can alter gamete viability and consequently seed yield (Gaeta \& Pires, 2010; Xiong et al., 2011). Selection of euploid individuals might thus, allow disentangling the consequences of aneuploidy from those of homoeolog rearrangements on meiotic behaviour and fertility.

So far, only rearrangements in the first generations after allopolyploidization have been studied in resynthesized $B$. napus without replicated lineages from the same SO and without distinguishing aneuploids from euploid individuals. Here, we aim to unravel the fate of these genomic exchanges in selected euploid generations of different resynthesized B. napus allopolyploids. By eliminating all aneuploid individuals, we can disregard the effect of aneuploidy on meiosis and seed production to focus on the structural and functional impact of homoeologous rearrangements. The extent and consequences 
of these homoeologous rearrangements in B. napus were followed in four resynthesized lines resulting from different genetic backgrounds, reciprocal parental crosses and different mode of allopolyploid formation. For each cross, several independent S1 lines were created in order to follow the dynamics of fixed homoeologous rearrangements over eight generations propagated by single seed descent (SSD). We assessed meiotic behaviour and fertility and explored the role of fixed non-reciprocal homoeologous exchanges on these traits in 358 individuals. Overall, our results highlight that selection of euploid individuals led to a strong decrease of newly fixed homoeologous rearrangements and disappearance of individuals with lethal rearrangements. We described homoeologous rearrangements having variable functional and structural impact on meiotic stability and seed production depending on the genetic background and cytoplasmic donor. We finally propose a model to explain genome stabilization process in natural B. napus.

\section{MATERIAL AND METHODS}

\section{Production of resynthesized B. napus populations through repeated selection of euploid individuals}

Resynthesized $B$. napus lines were created by crossing two different $B$. oleracea and $B$. rapa cultivars. For B. oleracea $(2 n=2 x=18, C C)$, we used the doubled haploid lines 'RC34' (B. oleracea var. alboglabra) and 'HDEM' (B. oleracea var. botrytis italica). For B. rapa ( $2 n=2 x=20, A A)$, we used 'Z1' (B. rapa var. trilocularis) and ' $\mathrm{C} 1.3$ ' (belonging to a fodder variety named 'chicon' var. rapifera). A resynthesized B. napus named 'RCC' was created by first crossing the B. oleracea 'RC34' (mother plant) and B. rapa 'C1.3' (father plant) lines, resulting in the amphiploid ' $F 1$ hybrid $R C C$ ' $(2 n=2 x=19, A C)$ (Fig. 1). This F1 hybrid was subsequently somatically doubled using colchicine (Chèvre et al. 1989), leading to the resynthesized B. napus 'RCC-SO' $(2 n=4 x=38)$. A reciprocal cross between the $B$. rapa ' $C 1 \cdot 3$ ' and the $B$. oleracea ' $\mathrm{RC} 34^{\prime}$ lines was also performed, leading to the amphiploid 'F1 hybrid CRC' and to the resynthesized B. napus 'CRC-SO' (after colchicine treatment). The two resynthesized 'RCC' and 'CRC' lines were selfed (hand pollination of floral buds before anthesis), producing the 'RCC-S1' and 'CRC-S1' progenies (Fig. 1). Thereafter 11 'RCC-S1' and 10 'CRC-S1' were selfed. These lineages were advanced by SSD until the eighth generation. At each generation, only one plant with 38 chromosomes (euploid) was randomly chosen from the set of euploid offspring. In some cases, lines did not produce any progeny, leading to the extinction of such lineage. In addition to ' $R C C$ ' and ' $C R C$ ', we produced resynthesized $B$. napus lines by firstly crossing the $B$. oleracea 'HDEM' line with the B. rapa ' $\mathrm{Z1}$ ' line. From this cross, we obtained three amphiploid F1 hybrids (named 'EMZ1', 'EMZ2' and 'EMZ3') that were somatically doubled using colchicine, producing the EMZ1-SO', 'EMZ2-SO' and 'EMZ3-SO' resynthesized lines. These three genetically identical EMZ lines were selffertilized, producing the 'EMZ1-S1', 'EMZ2-S1' and 'EMZ3-S1' progenies. Then, five to eight plants from 
each lineage were advanced by SSD until S8 generation (Fig. 1). The resynthesized 'UG EMZ' B. napus populations were produced by crossing the F1 hybrid 'EMZ1, 2 or 3' with the corresponding resynthesized B. napus 'EMZ1, 2 or 3 SO', leading to the formation 'UG EMZ1, 2 or 3 SO' B. napus lines. Resulting polyploids are thus the result of a cross between a female unreduced gamete of the F1 hybrid 'EMZ1' and a male reduced gamete of 'EMZ1-SO' (Fig. 1). After selfing these lines, 4 to 15 S1 plants from each line were advanced by SSD to the S8 generation.

\section{Meiotic behavior and chromosome counting}

The meiotic behavior and chromosome counting of all the produced material was studied by fixing floral buds in Carnoy's solution (ethanol-chloroform-acid acetic, 6:3:1) for 24 hours and then stored in 50\% ethanol. The anthers were squashed and stained in a drop of $1 \%$ acetocarmine solution: at least 20 pollen mother cells (PMCs) per plant were observed at metaphasis I.

\section{Fertility in resynthesized $B$. napus individuals}

The fertility (number of seeds per 100 pollinated flowers) of the different resynthesized B. napus was calculated by counting the number of pods per pollinated flowers at the bud stage (preventing the impact of potential self-incompatibility in the parental lines, as known for HDEM, Belser et al., 2018) and the number of seeds per pod allowing assessment of the number of seeds per pollinated flower. As a control, natural B. napus variety 'Darmor' was included in the experimental set-up.

\section{DNA extraction and SNP genotyping}

Genomic DNA of 157 individuals was extracted with the maxi plant kit (LGC Genomics, Teddington Middlesex, UK) at the GENTYANE platform (INRAE, France). For all four resynthesized crosses, only generations S0, S1, S3, S6 and S8 were genotyped. Specifically, DNA was extracted from 42 individuals for the 'RCC' resynthesized population including the two diploid parents, the $\mathrm{F} 1$ hybrid, 'RCC-SO', and 38 resynthesized progenies (11 S1, $11 \mathrm{~S} 3$, nine S6 and seven S8). For 'CRC', DNA from 38 individuals was extracted including the F1 hybrid, 'CRC-SO', and 36 resynthesized progenies (ten S1, nine S3, nine S6 and eight S8). For the different 'EMZ' populations, 61 individuals were submitted to DNA extractions; the diploid parents, three $\mathrm{F} 1$ hybrids, three 'EMZ-S0', and 50 resynthesized progenies (18 S1, 16 S3, nine S6 and seven S8). Finally, DNA extractions on resynthesized lines created via female unreduced gamete pathway were performed on 16 individuals from 'UG EMZ1-SO' cross, 43 individuals from 'UG EMZ2-S0' cross and 16 individuals from the 'UG EMZ3-S0' cross. 
Genotyping data were obtained using the Illumina (http://www.illumina.com/) Brassica 60K Infinium SNP array (Clarke et al., 2016) and were visualized with Genome Studio V2011.1 (Illumina, Inc., San Diego, CA, USA).

\section{SNP data analyses}

This Brassica 60K array is composed of 52,157 SNP markers that may either specifically hybridize to $B$. rapa or to $B$. oleracea or that may hybridize to both species. These latter markers thus hybridize to two distinct genome locations on $B$. napus (on the homoeologous $A$ and $C$ chromosomes). The positions of the SNP markers on the B. napus chromosomes derived from Rousseau-Gueutin et al., (2017) and were obtained by blasting the 52,157 sequence contexts (minimum of $90 \%$ global overlap and $90 \%$ identity) against the B. napus Darmor reference genome assembly (version 4.1 in Chalhoub et al., 2014). Only the markers presenting no more than one blast hit on each subgenome ( $A$ and $C$ ) were retained, enabling to discard SNPs potentially hybridizing at paralogous regions.

\section{Identification of deleted regions resulting from non-reciprocal homoeologous exchanges in each resynthesized $B$. napus line}

To identify non-reciprocal homoeologous exchanges in the 'RCC' and 'EMZ' resynthesized populations, we used two types of markers: i) homoeo-SNP markers (Mason et al., 2017) that were homozygous and

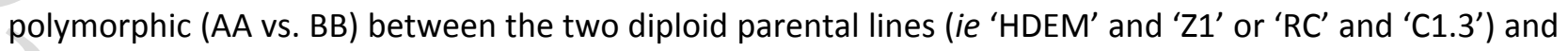
heterozygous in the SO B. napus and ii) 'dominant' markers that only hybridized in one diploid parental line of the $B$. napus resynthesized population (i.e. "AA" in 'HDEM' versus '--' in 'Z1'). Only the markers presenting identical genotype data for all four technical replicates were considered for further analyses. Thereafter, putative deletions in each resynthesized individual were identified by searching the loss of one parental allele in the polymorphic homoeo-SNP markers ('AB' in the SO and 'AA' or 'BB' in the resynthesized B. napus progenies) or in the dominant markers ('A-' or 'B-' in the SO and '--' in the resynthesized $B$. napus). To avoid false positive results, deleted regions were only considered if at least three consecutive markers displayed the loss of one parental allele (from the same parent). Additionally, within a genealogy, deletions (with identical start and end positions, or extended start and/or end positions) had to be inherited from parents to offspring. This method allows for the detection of deleted regions from fixed non-reciprocal homoeologous DNA exchange in resynthesized B. napus polyploids (Rousseau-Gueutin et al., 2017). To perform these analyses, we developed a SQL database using pgAdminIII (v1.22.1) that was saved in postgresql v9.5 (DataBase Management System). The size and gene content of each deleted region was thereafter determined. We also evaluated whether the deletions 
were present in the distal region of a chromosome arm (last $30 \%$ of a chromosome arm) or close to the pericentromere (Mason et al., 2016).

\section{Statistical analyses}

Multi-variable analyses and comparisons of means

In order to discriminate the different factors influencing both phenotyping (meiotic behavior and fertility) and genotyping variables (number, size and position of homoeologous rearrangements), we first conducted a Redundancy Analysis to summarize linear relationships between dependent variables and independent factors. Following this global analysis, statistical comparisons of means between crosses and between generations were performed using Anova and t-test with permutations when necessary. These statistical analyses and graphics were achieved using R language (R Core Team, 2018) and the RStudio environment (RStudio Team, 2020).

Probability distributions of typical measures of stability of meiosis and seed formation in newly resynthesized B. napus individuals

To statistically identify individual plants with extreme values of stability of meiosis and seed formation, we fitted probability density functions to the full cohort of observational data measured on the 358 resynthesized plants. The resulting probability distributions allowed weighting the probability of individual measure to conform or not to the expected pattern of meiosis and seed formation in newly resynthesized B. napus. Probability distributions were fitted using a classical approach of maximum likelihood estimate of parameters, by maximizing a log-likelihood function, with penalty applied for samples outside of range of the considered distribution. Considering the type and interval of measures, we fitted beta distributions for percentage measures (percentage of cells with 19 bivalents, with multivalents and percentage of male sterility), gamma distributions for mean positive measures (average number of univalent, bivalent and multivalent per PMC), binomial distributions for the ability to produce seeds and log-normal distribution for the number of seeds per 100 flowers.

\section{Genome scan for linking extreme phenotypic measures and deleted sites}

To identify putative genomic areas implied in the stability of meiosis and seed formation, we scanned the genome for deleted SNPs included in structural rearrangements (i.e deleted regions from one subgenome that most presumably result from homoeologous exchanges). We hypothesized that plants measured with extreme phenotypes may present specific deleted genomic areas involved in the stability of meiosis and seed formation. We thus classified for each SNP position, all studied plants in two groups: plants with 
or without this particular deleted SNP, and analyzed their phenotypic measures. We compared phenotypic measures of plant with and without deletions, and then computed the probability of overrepresentation of each identified deletion in plants with extreme phenotypic values.

In the first step, we tested if the two groups of plants, with and without deletion, differed for their phenotypic measures using classical Mann-Whitney rank tests. The advantage of such non-parametric test is to be less likely to find false significant differences than using parametric test, and thus allows identifying robust candidate regions. When such identified deletions occurred in different lineages with different genetic backgrounds, it strengthened our confidence that such deletion may include candidate genes involved in the stability of meiosis or seed formation. Indeed, different genetic backgrounds with the same deletion and the same phenotypic measures decrease the probability that identified extreme measures could be due to another deleted site co-inherited by descent from a common ancestor. We thus performed two complementary variations of this approach: one overall data and one within each genetic cross. First, we review each deletion regardless of their genetic background and tested if plants with deletion conformed to the standard distribution of phenotypic measures or not. Second, in each of the genetic combination, we tested if plants with deletion conformed to the standard distribution of phenotypic measures or not for each deletion, allowing to identify cross-specific loci that may be colocalized.

In the second step, we identified plants with extreme phenotypic measures when their measures lied outside the $99 \%$ confidence interval of each phenotypic measure, considering the appropriate fitted probability distributions (see above). Then, for each genomic site $i$, we counted the number of time $k_{i}$ this one was deleted among the $n_{i}$ abnormal plants. We computed the probability to observe $k_{i}$ deleted sites among $n_{i}$ by chance $P\left(k_{i} / n_{i}, p_{i}\right)$ as the probability mass function of a binomial distribution of success probability in each trial as the overall ratio of deleted sites on the number of successfully genotyped sites. A SNP candidate was considered as involved in the phenotype if its probability $P\left(k_{i} / n_{i}, p_{i}\right)$ was inferior to $10 \%$ in at least six individuals in different genetic backgrounds.

\section{RESULTS}

The dataset presented here comprises phenotypic measures of SDD individuals over eight generations for four independent nascent lineages of allopolyploid B. napus. The impact of repetitive euploid selection was visible as soon as the fourth generation and forward, as only 0 to $2.94 \%$ of aneuploid individuals were found in the resynthesized S4 to S8 generations compared to $11.46-11.27 \%$ in S1-S3 (Supporting Information Fig. S1). In total, we assessed the meiotic behavior and seed yield of 358 individuals including 
73 individuals for each 'RCC' and CRC' lines, as well as 91 and 121 'EMZ' and UG EMZ' individuals, respectively. All individual measurements included number of seeds per 100 flowers, percentages of cells with multivalents and bivalents as well as, mortality in the next generation (Supporting Information Table S1). This valuable dataset was then analysed in regards to a genotyping dataset performed on the four lines at generation S1, S3, S6 and S8. Each resynthesized B. napus lineage is represented by 35, 37, 40 and 62 'RCC', 'CRC', 'EMZ' and 'UG EMZ' individuals, respectively. Description (number, size, position) of the identified homoeologous rearrangements was included in Supporting Information Table S1. Overall, variability of the dataset was well explained $(68 \%, p<0.001)$ by the factors 'cross' and 'generation', as well as by the interaction of 'cross' and 'generation' ( $p<0.001$; Supporting Information Table S2). We thus mined the datasets to identify the factors explaining the phenotypic and genomic variations between genetic backgrounds as well as the dynamics in the first eight generations after allopolyploid speciation.

\section{Fertility}

The fertility of the resynthesized $B$. napus populations was assessed based on the number of seeds per 100 flowers in all four nascent lines of $B$. napus 'RCC', 'CRC', 'EMZ' and 'UG EMZ'. Means for the nascent lines were 270.8, 158.8, 62.6 and 55.1, respectively. By comparison, variety 'Darmor' has on average 2067 seeds per 100 flowers ( $S D=516)$, which is significantly 10-fold higher than what is observed in resynthesized B. napus (t-test with permutation, $\mathrm{p}=0.0025$, Supporting Information Fig. S2). Overall, differences in seed yield were significant between all lines ( $t$-test, $p<0.01$ ) except between 'EMZ' vs 'UG EMZ' (Supporting Information Fig. S2). In 'RCC', fertility significantly decreased from the $1^{\text {st }}$ generation to the $4^{\text {th }}(\mathrm{t}$-test, $\mathrm{p}<0.01)$ and again from the $5^{\text {th }}$ to the $8^{\text {th }}$ generation $(\mathrm{t}$-test, $\mathrm{p}<0.01)$ with a high fertility (mean=572.2) observed in the $5^{\text {th }}$ generation (comparable to the yield observed in S1) (Fig. 2). In 'CRC', fertility increased in the 2 nd generation compared to the first generation (162.5 to 395.8 seeds per flower, $p<0.05$ ) to drastically decrease until the $8^{\text {th }}$ generation (t-test, $p<0.05$ ) (Fig. 2). In 'EMZ', compared to SO all following generations presented a lower number of seeds. Similarly, 'UG EMZ' individuals exhibited equal or lower number of seeds in the generations following allopolyploidization, only a slight significant decrease was observable from generation S1-S2 to S3 (t-test, $p<0.05$ ) (Fig. 2).

\section{Meiotic behavior}

Meiotic behavior was characterized using various descriptors. First, as solely euploid B. napus individuals (with $2 n=38$ ) were assessed, it was expected that such plants presented a better meiotic stability compared to its aneuploid siblings. Despite having 38 chromosomes, meiosis was affected in our plants with $49.4 \%, 41.0 \%, 41.1$ and $38.1 \%$ of cells exhibiting 19 bivalent structures in 'RCC', 'CRC', 'EMZ' and 'UG 
EMZ', respectively overall generations (Supporting Information Fig. S2). Percentage of cells with 19 bivalents was significantly higher in 'RCC' compared to the three other nascent lines (Supporting Information Fig. S2, t-test, $\mathrm{p}<0.01)$.

SO individuals in all nascent lines exhibited overall higher percentages of cells with proper bivalents (80.0\% in RCC, $75.0 \%$ in CRC, $71.6 \%$ in EMZ and $76.3 \%$ in UG EMZ) compared to subsequent generations. Percentage of cells with 19 bivalents was consistent across generations S1 to S8 in 'RCC' whereas significant differences between generations were visible in the crosses 'CRC', 'EMZ' and 'UG EMZ' (Fig. 2). In 'CRC', percentage of cells with 19 bivalents tended to increase in S3 compared to S1 (from $21.1 \%$ to $44.8 \%$, t-test, $p<0.05$, Fig. 2) and stabilized in subsequent generations. In 'EMZ', percentage of cells with 19 bivalents decreased drastically from S1 to S2 (t-test, $p<0.05$, Fig. 2) followed by a slight increase from S2 to S7. In 'UG EMZ', after the drop from SO to S1, percentage of cells with 19 bivalents was found constant with a slight increase in S8 (Fig. 2).

Finally, we assessed the percentage of cells exhibiting multivalents in the nascent allopolyploids. A similar percentage of cells with multivalents was observed among all lines $(27.3 \%$ in ' $R C C$ ', $28.8 \%$ in 'CRC', $29.7 \%$ in 'EMZ' and $29.1 \%$ in 'UG EMZ', Supporting Information Fig. S2). This trend was also found consistent across generations for all resynthesized lines except for 'UG EMZ' where a slight increase in the percentage of multivalents was observed from S1 to S4-S5 (23.4\% to $34.7 \%$, t-test, p<0.05, Fig. 2). After that, the predominance of multivalents decreased from $\mathrm{S} 5$ to $\$ 8$.

\section{Identification of fixed non reciprocal structural rearrangements}

To identify the putative presence of fixed homoeologous rearrangements in each genotype, we used the polymorph markers between the diploid parental lines of resynthesized B. napus: 12,218 markers for 'RCC' and 'CRC' (on average one every 68.8kb) and 15,180 markers for 'EMZ' and 'UG EMZ' (one marker every $55.3 \mathrm{~kb})$.

We then evaluated size, number and position on the chromosomes and on the subgenomes of fixed homoeologous rearrangements in a population per resynthesized line and per generation (Supporting Information Table S1), using the SNP physical localization (Rousseau-Gueutin et al., 2017). Average number of identified regions ranged between 5.2, 6.1, 8.1 and 11.2 per individual per generation in 'RCC', in 'CRC', in 'EMZ' and 'UG EMZ' respectively (Fig. 3). Globally, individuals from the 'UG EMZ' line showed significantly higher number of rearranged regions than ' $C R C$ ' and ' $R C C^{\prime}$ lines (t-test, $p<0.01$, Fig. 3). The average size of the homoeologous rearrangements was estimated at 1.88, 3.79, 2.43 and $3.06 \mathrm{Mb}$ in ' $\mathrm{RCC}$ ', in 'CRC', in 'EMZ' and 'UG EMZ' respectively (Fig. 3). A significant difference was only observed between 'RCC' and 'UG EMZ' (t-test, p<0.01, Fig. 3). 
We observed a limited number of homoeologous rearrangements in ' $R C C^{\prime}$ ' and 'CRC' compared to other crosses, with the exception of one 'CRC' individual in S6 and S8 (Fig. 3). By contrast, number of homoeologous rearrangements is higher as soon as the S1 generation in 'UG EMZ' and to a lesser extent in 'EMZ'. We observed a low number of individuals in generations 58 in both 'EMZ' and 'UG EMZ' compared to ' $\mathrm{RCC}$ ' and ' $\mathrm{CRC}$ '. Interestingly, by decomposing the nature of these homoeologous rearrangements for each individual compared to previous generation, we could infer the drastic decrease of new homoeologous rearrangements appearing in the different lineages at S3 for 'RCC' and at S6 for 'CRC', 'EMZ' and 'UG EMZ' (Supporting Information Fig. S3).

In parallel, we assessed cumulative size of homoeologous rearrangements on each subgenome from generation S1 to S8 in all crosses (Fig. 4). Overall, cumulative size of homoeologous rearrangements was found higher in 'UG EMZ' compared to 'EMZ' and 'RCC' (with $35.2 \mathrm{Mb}$ vs $20.5 \mathrm{Mb}$ and $12.4 \mathrm{Mb}$; t-test, $\mathrm{p}<0.05)$ (Fig. 4). Furthermore, the $\mathrm{C}$ subgenome was predominantly affected by rearrangements in 'CRC', 'EMZ' and 'UG EMZ' (Fig. 4) but was only statistically different in generations S6 and S8 of 'CRC', generation S3 of 'EMZ' and S3 of 'UG EMZ'. Although having similar progenitors, crosses 'RCC' and CRC' were found differently impacted by homoeologous rearrangements on the C subgenomes in S6 and S8 ( $t$ test, $\mathrm{p}<0.05)$.

\section{Correlations between homoeologous rearrangements, meiotic behaviour and fertility}

The novelty of the present study lies in explaining meiotic behavior, fertility, and structural dynamics using repeated euploid selection of $B$. napus individuals in nascent allopolyploid populations. We assessed correlations between the different variables in order to identify if and how fixed non-reciprocal translocations may explain seed yield and chromosome pairing during meiosis. First, all measurements describing the presence of homoeologous rearrangements were correlating positively between themselves (Supporting Information Fig. S4). Expectedly, percentage of cells with bivalents was inversely correlated with percentage of cells with multivalents (with statistical support in crosses 'RCC', 'EMZ' and 'UG EMZ', Supporting Information Fig. S4). Interestingly, the correlations depended profoundly on the cross and thus the progenitors of the resynthesized allopolyploids. In 'RCC' and 'CRC' that have globally fewer homoeologous rearrangements, strongest negative correlations were found between the presence of homoeologous rearrangements and the number of seeds produced (Supporting Information Fig. S4). By contrast, large and numerous rearrangements observed in 'EMZ' have strong negative impact on meiotic behavior (Supporting Information Fig. S4). In 'UG EMZ', correlations were described between average size of rearrangements and meiotic behavior and between number of homoeologous rearrangements along with cumulative size on A subgenome and fertility (Supporting Information Fig. S4). 


\section{Genome scan linking phenotypic measures with rearranged homoeologous regions}

To go further, a genome scan was performed to link phenotypic measures with positions of the rearranged homoeologous regions (Supporting Information Table S3). Using genome scan analysis overall data, we identified one locus of $2.2 \mathrm{Mb}$ on chromosome A03 containing 440 genes (Table 1 ) and impacting the percentage of cells with multivalents. This list of genes was screened to identify annotations that might be relevant in the context of plant meiosis. In particular, one gene (ortholog AT2G42890) coding for a protein MEI2-like 2 or ML2 has drawn our attention. We looked at copy number variation of this gene in the parental lines of the resynthesized B. napus populations 'EMZ' and 'UG EMZ' (B. rapa cV. Z1 and B. oleracea cv. HDEM) for which whole genome assemblies are available (Belser et al., 2018). We observed that this gene was present in two intact copies in both species (on A03 and A05 for B. rapa, C03 and C04 for $B$. oleracea) and that these copies are in orthologous regions ( $\mathrm{A03} / \mathrm{CO3}, \mathrm{A} 05 / \mathrm{CO} 4)$. Using genome scan analyses within each cross, we identified one region associated with the percentage of cells with 19 bivalents in 'EMZ' and 'CRC' individuals on the C02 chromosome and three regions associated with the number of seeds per 100 flowers on C01, C02 and C04 chromosomes (containing 228 to 880 genes, Table 1).

\section{DISCUSSION}

In this study, we unravel the consequences of repeated euploid selection in the early generations following allopolyploidization, specifically we describe how rearrangements between homoeologous chromosomes influence meiotic behavior and fertility in nascent resynthesized $B$. napus individuals. Compared to previous studies using resynthesized B. napus (Gaeta \& Pires, 2007; Szadkowski et al., 2010; Xiong \& Pires, 2011; Xiong et al., 2011; Rousseau-Gueutin et al., 2017; Bird et al., 2020), we decided to perform repetitive selection for euploid individuals in order to evaluate the role of this phenomenon in $B$. napus speciation success. Ultimately, after a few euploid generations the consequences on meiosis and fertility can be attributed primarily to homoeologous rearrangements thus drawing conclusion on the impact of homoeologous rearrangements on genome stabilization. After the first meiosis and its 'genome blender', we focus on the drastic subsequent increase of homoeologous rearrangements from S1 to S4, rapidly followed by a decrease in number of newly fixed homoeologous rearrangements in all studied resynthesized B. napus lineages. Interestingly, their consequences on meiotic behavior and seed number strongly depended on the genetic background and cytoplasm donor. Finally, we discuss the origin of $B$. napus and the processes that could explain their genomic stability compared to resynthesized allopolyploids. 


\section{Impact of the first meiosis depends on the different genetic backgrounds}

Immediately after the formation of the different resynthesized B. napus lines, S0 individuals exhibited a relatively high percentage of cells with bivalents and low percentage of cells with multivalents due to the absence of rearrangements (Szadkowski et al., 2010, 2011). In the S1 generation, percentage of cells with 19 bivalents significantly decreased while homoeologous rearrangements were fixed, favoring subsequent homoeologous pairing notably via the formation of multivalents.

The extent of homoeologous rearrangement intensification in the first couple of generations after allopolyploid speciation depends on the genetic background. Number of homoeologous rearrangements and cumulative size in S3 is strongly limited in 'RCC' and 'CRC' compared to 'EMZ'. These differences can be explained by the contrasted genetic backgrounds of resynthesized B. napus progenitors. First, macrosyntenic differences (large structural variants) between progenitor genomes could be present leading to different rates of homoeologous pairing and crossing-over in the allopolyploid. Both Brassica rapa used in this study (' $\mathrm{Z} 1$ ' ssp trilocularis and 'C1.3' ssp rapifera) and both B. oleracea progenitors ('HDEM' ssp botrytis 'broccoli' type vs 'RC34' ssp alboglabra 'chinese kale') come from distinct clades (Cheng et al., 2016). Although, the genome structure of ' $\mathrm{C} 1.3$ ' and 'RC34' is still unknown, a previous RNASeq study on the same resynthesized polyploids demonstrated the divergence in terms of transcription between progenitors and different transcriptional dynamics in the allopolyploids (Ferreira de Carvalho et al., 2019). Globally, large structural diversity is being described within both $B$. rapa and B. oleracea species (Lin et al., 2014; Golicz et al., 2016; Boutte et al., 2020) and could participate in the variation in number and size of homoeologous rearrangements. Second, presence of a genetic factor controlling meiotic behavior in allopolyploids and preventing homoeologous pairing could also explain the differences in the number of rearrangements observed and in the percentage of cells with bivalents between 'RCC' and 'EMZ'. One approach consisted in correlating the loss of one homoeologous region with the variation observed in the different phenotypic variables. We notably identified one region on the A03 chromosome containing 440 genes in 'RCC', 'CRC' and 'UG EMZ' and leading to fewer cells with multivalents. Interestingly, one meiotic gene is included in this region and has been shown implicated in meiosis and control of chromosome pairing. ML2 gene has been shown to play a role in fertility and plant meiotic behavior in A. thaliana mutants (Kaur et al., 2006). In RNAi triple mutants for am/1, am/2 and am/4, phenotypes observed were due to a range of abnormalities in chromosome organization during meiotic prophase and later stages. Here, resynthesized lines of $B$. napus were not subjected to a complete loss of the locus but the replacement of the $\mathrm{A} 03$ region by the homoeologous region from the $\mathrm{CO} 3$ chromosome 
subgenome. Thus, as the copy number of this gene did not vary between $B$. napus resynthesized lines, the statistically different number of cells with multivalents between some individuals may result from allelic or different transcript levels of the A03 vs C03 copy. Similarly, other genes have been described as implicated in homoeologous pairing control via allele or dosage effect, such as MSH4 (Gonzalo et al., 2019). BnaPH1 and PrBn have additionally been shown to reduce homoeologous pairing (Jenczewski et al., 2003; Higgins et al., 2021).

Finally, with identical diploid progenitors, resynthesized individuals 'EMZ' and 'UG EMZ' exhibited similar fertility and meiotic behavior but showed contrasted patterns regarding their respective cumulative size of fixed homoeologous rearrangements. These two resynthesized allopolyploids differ only by their mode of polyploid formation. The SO from 'EMZ' has been produced via colchicine treatment whereas 'UG EMZ' SO has been produced by unreduced gametes following the hybridization of one 'EMZ' SO and one 'EMZ' F1 hybrid. Unreduced gametes formed in allohaploids $(n=2 x=19 ; A C)$ present an elevated frequency of homoeologous exchanges during meiosis compared to allotetraploids $B$. napus ( $2 n=4 X=38$; AACC) (Nicolas et al., 2007, 2009, 2012; Cifuentes et al., 2010; Szadkowski et al., 2011). Indeed, 'UG EMZ' S3 individuals, cumulating rearrangements generated from unreduced female gametes with the ones occurring in SO synthetic male gamete, have two to three-fold higher number of rearrangements and larger cumulative size than 'EMZ' S3 individuals. However, this tendency diminishes in S6 and S8 in 'UG EMZ' individuals thanks to the low fixation of novel homoeologous rearrangements over generation via the recurrent selection of euploid individuals as well as the high mortality rate of individuals with numerous rearrangements.

\section{Consequences of euploid selection on the fixation rate of new homoeologous rearrangements in} early allopolyploid generations

Rapidly after the third generation, euploid selection led to the fixation of fewer novel homoeologous rearrangements hence, limiting the negative impact of these rearrangements on genome stability and seed yield. Indeed, in accordance with previous studies demonstrating the consequences of aneuploidy (Gaeta \& Pires, 2010; Xiong et al., 2011) we confirmed experimentally how euploid selection fostered higher or at least constant number of bivalents during meiosis, the amplitude being dependent on the genetic background and maternal progenitor of the resynthesized allopolyploids. However, in our case, no significant improvement of seed yield was observed over the first eight generations. 
The efficiency of the genome blender is determinant for the meiotic stability in advanced generations. 'RCC' had fewer, shorter rearrangements without improved seed yield in S8, which probably reveals the functional impact of homoeologous rearrangements on this trait. As numerous seed yield QTLs were identified along the genome of $B$. napus (Raboanatahiry et al., 2018), we can presume that even few rearrangements in those regions will have an impact on the production of seeds. In this case, the decrease in seed number may be linked to the allelic diversity and/or dosage effect of genes present in the homoeologous rearrangements. On the other hand, 'CRC' demonstrates fewer and shorter rearrangements with poorer seed yield than 'RCC'. With identical genetic backgrounds, both crosses should be similarly functionally impacted by homoeologous rearrangements on seed production. Interestingly, 'CRC' individuals have additional homoeologous rearrangements on the $\mathrm{C}$ subgenome in generation $\mathrm{S} 6$ and $\mathrm{S} 8$ compared to 'RCC'. This observable difference between 'RCC' and 'CRC is probably due to the maternal donors. Phenotypic consequences of differential cytoplasm donors have been reported in Brassica (Cui et al., 2012) and in tomato (Demondes de Alencar et al., 2020) and recently in maternal cross combination of resynthesized B. napus (Sosnowska et al., 2020).

In both 'EMZ' and 'UG EMZ', large and numerous homoeologous rearrangements were observed, directly influencing the stability of meiosis and decreasing the percentage of cells with 19 bivalents. In this case, having smaller and fewer homoeologous rearrangements improved meiotic stability but not seed production as observed in 'RCC' and to a lesser extent in 'CRC'. Although the number of new homoeologous rearrangements seemed to be limited as soon as the 6th generation, individuals still carry a large deleterious load on fertility and meiotic behavior that would not be purged in these self-fertilized lines. Hence, these fixed homoeologous rearrangements may only be eliminated from the population by extensive outcrossing events.

\section{On the road to polyploid success}

Homoeologous rearrangements are visible in B. napus varieties (Chalhoub et al., 2014; Lloyd et al., 2018; Song et al., 2020). These rearrangements are globally shorter in natural varieties compared to resynthesized lines (Chalhoub et al., 2014) and do not seem to influence meiotic behavior and fertility (Rousseau-Gueutin et al., 2017). Recently, the pangenome of B. napus revealed the high proportion of homoeologous genomic rearrangements in modifying important adaptive and agronomic traits, such as flowering time, seed quality, silique length, disease resistance and chemical defense (Pires et al., 2004; Zhao et al., 2006; Hurgobin et al., 2017; Stein et al., 2017; Song et al., 2020). It is thus obvious that these rearrangements are occurring and may sometime be beneficial to agronomically improve $B$. napus 
varieties. Yet, these homoeologous rearrangements observed in natural B. napus never level up to the number and size of homoeologous rearrangements observed in newly resynthesized $B$. napus lines (Szadowski et al., 2010, 2011; Rousseau-Gueutin et al., 2017). Thus, we can hypothesize that the original B. napus population has experienced similar homoeolog rearrangements as observed in the resynthesized allotetraploids, but that overlapping between rearrangements of different sizes in the same genomic region allowed crossover formation leading to progressive size decrease of homoeologous rearrangements (in the case of intercrossing). Thereby, the current B. napus varieties most presumably derive from a small founding population of euploid and fertile individuals that intercrossed to minimize the number of fixed homoeologous rearrangements, hence maximizing the number of bivalent and enhancing seed production. The alternative (or complementary) explanation lies in the presence of a genetic determinant that completely or partially prevented homoeologous pairing and rearrangements, and preexisted in B. napus diploid progenitors.

\section{CONCLUSION}

To conclude, the consequences of homoeologous rearrangements on meiotic behavior and fertility depends on their size and the genetic background where they occur. Although recurrent euploid selection reduced the fixation rate of novel homoeologous rearrangements in subsequent generations, it did not directly improve fertility and meiotic stability. Interestingly, the rearrangements identified in these $B$. napus enabled the identification of several candidate regions involved in seed yield and genome stability. These results offer a new perspective on the consequences of structural variants in allopolyploid genome stability and speciation success as well as new avenues to increase phenotypic diversity in oilseed rape.

\section{ACKNOWLEDGEMENTS}

This work was made possible by financial support of the European Union, a Marie Sklodowska-Curie grant (number: 791908) awarded to JFdC, and a grant from the department 'Biology and Plant Breeding' at INRAE (awarded to MR-G). We acknowledge the BrACySol BRC (INRA Ploudaniel, France) that provided us with the seeds of parental lines; and K. C. Falk from Agriculture and Agri-Food Canada (Ontario) that provided the seeds of $B$. rapa ' $Z 1$ '. We would also like to thank all the technical staff of the greenhouse for management of the plant material (especially L. Charlon, P. Rolland, J.-P. Constantin, J.-M. Lucas and F. Letertre) and G. Bianchetti for essential input on Rstudio and ggplot2 package. The authors have no conflict of interest to declare.

\section{AUTHOR CONTRIBUTION}


A-MC was involved in the experimental design with JFdC and MR-G in the conceptualization of the study; FE, ML-T, GT carried out the molecular and cytological experiments; M-MG was in charge of plant care; JFdC, SS, JM, CF, A-MC, MRG were involved in data analyses; JFdC, SS, A-MC and MR-G were involved in writing.

\section{REFERENCES}

An H, Qi X, Gaynor ML, Hao Y, Gebken SC, Mabry ME, McAlvay AC, Teakle GR, Conant GC, Barker MS, et al. 2019. Transcriptome and organellar sequencing highlights the complex origin and diversification of allotetraploid Brassica napus. Nature Communications 10: 2878.

Anderson GH, Hanson MR. 2005. The Arabidopsis Mei2 homologue AML1 binds AtRaptor1B, the plant homologue of a major regulator of eukaryotic cell growth. BMC plant biology 5: 2 .

Baniaga AE, Marx HE, Arrigo N, Barker MS. 2020. Polyploid plants have faster rates of multivariate niche differentiation than their diploid relatives. Ecology Letters 23: 68-78.

Belser C, Istace B, Denis E, Dubarry M, Baurens F-C, Falentin C, Genete M, Berrabah W, Chèvre A-M, Delourme R, et al. 2018. Chromosome-scale assemblies of plant genomes using nanopore long reads and optical maps. Nature Plants 4: 879-887.

Bird KA, Niederhuth C, Ou S, Gehan M, Pires JC, Xiong Z, VanBuren R, Edger PP. 2020. Replaying the evolutionary tape to investigate subgenome dominance in allopolyploid Brassica napus. New Phytologist doi.org/10.1111/nph.17137.

Boutte J, Maillet L, Chaussepied T, Letort S, Aury J-M, Belser C, Boideau F, Brunet A, Coriton O, Deniot G, et al. 2020. Genome size variation and comparative genomics reveal intraspecific diversity in Brassica rapa. Frontiers in Plant Science 11: 577536.

Chalhoub B, Denoeud F, Liu S, Parkin IAP, Tang H, Wang X, Chiquet J, Belcram H, Tong C, Samans B, et al. 2014. Early allopolyploid evolution in the post-Neolithic Brassica napus oilseed genome. Science $\mathbf{3 4 5}$ : 950-953.

Cheng F, Wu J, Cai C, Fu L, Liang J, Borm T, Zhuang M, Zhang Y, Zhang F, Bonnema G, et al. 2016. Genome resequencing and comparative variome analysis in a Brassica rapa and Brassica oleracea collection. Scientific Data 3: 160119.

Cifuentes M, Eber F, Lucas M-O, Lode M, Chèvre A-M, Jenczewski E. 2010. Repeated polyploidy drove different levels of crossover suppression between homoeologous chromosomes in Brassica napus allohaploids. The Plant Cell 22: 2265-2276. 
Cui C, Ge X, Gautam M, Kang L, Li Z. 2012. Cytoplasmic and genomic effects on meiotic pairing in brassica hybrids and allotetraploids from pair crosses of three cultivated diploids. Genetics 191: 725-738.

Delmondes de Alencar L, Azevedo P, Rocha Latado R. 2020. Mothers' command: phenotypes changes resulting from reciprocal interploidy crosses. Euphytica 216: 21.

Ferreira de Carvalho J, Lucas J, Deniot G, Falentin C, Filangi O, Gilet M, Legeai F, Lode M, Morice J, Trotoux G, et al. 2019. Cytonuclear interactions remain stable during allopolyploid evolution despite repeated whole-genome duplications in Brassica. The Plant Journal 98: 434-447.

Gaeta RT, Pires CJ. 2010. Homoeologous recombination in allopolyploids: the polyploid ratchet. New Phytologist 186: 18-28.

Gaeta RT, Pires JC, Iniguez-Luy F, Leon E, Osborn TC. 2007. Genomic changes in resynthesized Brassica napus and their effect on gene expression and phenotype. Plant Cell 19: 3403-3417.

Golicz AA, Bayer PE, Barker GC, Edger PP, Kim H, Martinez PA, Chan CKK, Severn-Ellis A, McCombie WR, Parkin IAP, et al. 2016. The pangenome of an agronomically important crop plant Brassica oleracea. Nature Communications 7: 13390.

Gonzalo A, Lucas M-O, Charpentier C, Sandmann G, Lloyd A, Jenczewski E. 2019. Reducing MSH4 copy number prevents meiotic crossovers between non-homologous chromosomes in Brassica napus. Nature Communications 10: 2354.

Griffiths S, Sharp R, Foote TN, Bertin I, Wanous M, Reader S, Colas I, Moore G. 2006. Molecular characterization of $P h 1$ as a major chromosome pairing locus in polyploid wheat. Nature 439: 749-752.

Higgins EE, Clarke WE, Howell EC, Armstrong SJ, Parkin IAP. 2018. Detecting de novo homoeologous recombination events in cultivated Brassica napus using a genome-wide SNP array. G3: Genes, Genomes, Genetics 8: 2673-2683.

Higgins EE, Howell EC, Armstrong SJ, Parkin IAP. 2021. A major quantitative trait locus on chromosome A9, BnaPh1, controls homoeologous recombination in Brassica napus. New Phytologist 229: 3281-3293. Hurgobin B, Golicz AA, Bayer PE, Chan C-KK, Tirnaz S, Dolatabadian A, Schiessl SV, Samans B, Montenegro JD, Parkin IAP, et al. 2018. Homoeologous exchange is a major cause of gene presence/absence variation in the amphidiploid Brassica napus. Plant Biotechnology Journal 16: 12651274.

Jenczewski E, Alix K. 2004. From diploids to allopolyploids: the emergence of efficient pairing control genes in plants. Critical Reviews in Plant Sciences 23: 21-45.

Jenczewski E, Eber F, Grimaud A, Huet S, Lucas MO, Monod H, Chèvre AM. 2003. PrBn, a major gene controlling homeologous pairing in oilseed rape (Brassica napus) haploids. Genetics 164: 645-653. 
Jesske T, Olberg B, Schierholt A, Becker HC. 2013. Resynthesized lines from domesticated and wild Brassica taxa and their hybrids with B. napus L.: genetic diversity and hybrid yield. TAG Theoretical and applied genetics 126: 1053-1065.

Jiao Y, Wickett NJ, Ayyampalayam S, Chanderbali AS, Landherr L, Ralph PE, Tomsho LP, Hu Y, Liang H, Soltis PS, et al. 2011. Ancestral polyploidy in seed plants and angiosperms. Nature 473: 97-100.

Lin K, Zhang N, Severing El, Nijveen H, Cheng F, Visser RG, Wang X, de Ridder D, Bonnema G. 2014. Beyond genomic variation - comparison and functional annotation of three Brassica rapa genomes: a turnip, a rapid cycling and a Chinese cabbage. BMC Genomics 15: 250.

Lloyd A, Blary A, Charif D, Charpentier C, Tran J, Balzergue S, Delannoy E, Rigaill G, Jenczewski E. 2018. Homoeologous exchanges cause extensive dosage-dependent gene expression changes in an allopolyploid crop. The New Phytologist 217: 367-377.

Mason AS, Higgins EE, Snowdon RJ, Batley J, Stein A, Werner C, Parkin IAP. 2017. A user guide to the Brassica 60K Illumina Infinium ${ }^{\mathrm{TM}}$ SNP genotyping array. TAG Theoretical and applied genetics 130: 621633.

Mason AS, Rousseau-Gueutin M, Morice J, Bayer PE, Besharat N, Cousin A, Pradhan A, Parkin IAP, Chèvre A-M, Batley J, et al. 2016. Centromere locations in Brassica $A$ and $C$ genomes revealed through half-tetrad analysis. Genetics 202: 513-523.

Nicolas SD, Leflon M, Monod H, Eber F, Coriton O, Huteau V, Chèvre A-M, Jenczewski E. 2009. Genetic regulation of meiotic cross-overs between related genomes in Brassica napus haploids and hybrids. The Plant Cell 21: 373-385.

Nicolas SD, Mignon GL, Eber F, Coriton O, Monod H, Clouet V, Huteau V, Lostanlen A, Delourme R, Chalhoub B, et al. 2007. Homeologous recombination plays a major role in chromosome rearrangements that occur during meiosis of Brassica napus haploids. Genetics 175: 487-503.

Nicolas SD, Monod H, Eber F, Chèvre A-M, Jenczewski E. 2012. Non-random distribution of extensive chromosome rearrangements in Brassica napus depends on genome organization. The Plant Journal 70: 691-703.

One thousand plant transcriptomes. 2019. One thousand plant transcriptomes and the phylogenomics of green plants. Nature 574: 679-685.

Pires CJ, Zhao J, Schranz ME, Leon E, Quijada PA, Lukens LN, Osborn TC. 2004. Flowering time divergence and genomic rearrangements in resynthesized Brassica polyploids (Brassicaceae). Biological Journal of the Linnean Society 82: 675-688.

R Core Team. 2018. R: A Language and Environment for Statistical Computing. Vienna, Austria: $\mathrm{R}$ Foundation for Statistical Computing. 
Raboanatahiry N, Chao H, Dalin H, Pu S, Yan W, Yu L, Wang B, Li M. 2018. QTL alignment for seed yield and yield related traits in Brassica napus. Frontiers in Plant Science $9: 1127$.

Rousseau-Gueutin M, Morice J, Coriton O, Huteau V, Trotoux G, Nègre S, Falentin C, Deniot G, Gilet M, Eber $\mathbf{F}$, et al. 2017. The impact of open pollination on the structural evolutionary dynamics, meiotic behavior, and fertility of resynthesized allotetraploid Brassica napus L. G3: Genes, Genomes, Genetics 7: 705-717.

RStudio Team. 2020. RStudio: Integrated Developement for R. RStudio, PBC, Boston, MA URL http://www.rstudio.com/ last accessed November 2020.

Samans B, Chalhoub B, Snowdon RJ. 2017. Surviving a genome collision: genomic signatures of allopolyploidization in the recent crop species Brassica napus. The Plant Genome 10 (3) plantgenome2017.02.0013.

Selmecki AM, Maruvka YE, Richmond PA, Guillet M, Shoresh N, Sorenson AL, De S, Kishony R, Michor F, Dowell R, et al. 2015. Polyploidy can drive rapid adaptation in yeast. Nature 519: 349-352.

Song J-M, Guan Z, Hu J, Guo C, Yang Z, Wang S, Liu D, Wang B, Lu S, Zhou R, et al. 2020. Eight highquality genomes reveal pan-genome architecture and ecotype differentiation of Brassica napus. Nature Plants 6: 34-45.

Song K, Lu P, Tang K, Osborn TC. 1995. Rapid genome change in synthetic polyploids of Brassica and its implications for polyploid evolution. Proceedings of the National Academy of Sciences of the United States Of America 92: 7719-7723.

Sosnowska K, Majka M, Majka J, Bocianowski J, Kasprowicz M, Książczyk T, Szała L, Cegielska-Taras T. 2020. Chromosome instabilities in resynthesized Brassica napus revealed by FISH. Journal of Applied Genetics 61: 323-335.

Szadkowski E, Eber F, Huteau V, Lode M, Coriton O, Jenczewski E, Chevre AM. 2011. Polyploid formation pathways have an impact on genetic rearrangements in resynthesized Brassica napus. New Phytologist 191: 884-894.

Szadkowski E, Eber F, Huteau V, Lode M, Huneau C, Belcram H, Coriton O, Manzanares-Dauleux MJ, Delourme R, King GJ, et al. 2010. The first meiosis of resynthesized Brassica napus, a genome blender. New Phytologist 186: 102-112.

Tate JA, Ni Z, Scheen AC, Koh J, Gilbert CA, Lefkowitz D, Chen ZJ, Soltis PS, Soltis DE. 2006. Evolution and expression of homeologous loci in Tragopogon miscellus (Asteraceae), a recent and reciprocally formed allopolyploid. Genetics 173: 1599-1611. 
Xiong Z, Gaeta RT, Pires JC. 2011. Homoeologous shuffling and chromosome compensation maintain genome balance in resynthesized allopolyploid Brassica napus. Proceedings of the National Academy of Sciences of the United States Of America 108: 7908-7913.

Xiong Z, Pires JC. 2011. Karyotype and identification of all homoeologous chromosomes of allopolyploid Brassica napus and its diploid progenitors. Genetics 187: 37-49.

Zhao J, Udall JA, Quijada PA, Grau CR, Meng J, Osborn TC. 2006. Quantitative trait loci for resistance to Sclerotinia sclerotiorum and its association with a homeologous non-reciprocal transposition in Brassica napus L. TAG Theoretical and applied genetics 112: 509-516.

Legends to Supporting Information

Fig. S1 Impact of artificial euploid selection

Fig. S2 Differences in fertility and meiotic behavior among resynthesized B. napus populations.

Fig. S3 Evolution of structural rearrangements across all generations.

Fig. S4 Correlations between variables in all four resynthesized B. napus populations.

Table S1 Data table of phenotypic measures and structural rearrangements.

Table S2 Results of redundancy analysis.

Table S3 Results of the genome scan analysis. 


\section{FIGURE LEGENDS}

Figure 1. Crosses and gamete type formation of the different resynthesized Brassica napus populations advanced by single seed descent.

Figure 2. Evolution of number of seeds per 100 flowers, percentage of cells with 19 bivalents and percentage of cells with multivalents during Metaphasis I in all four resynthesized Brassica napus populations across all generations. Boxplots represent interquartile range, horizontal line, the median with minimum and maximum values in the data. Letters above represent significativity after t-test $(p<0.05)$.

Figure 3. Structural dynamics of fixed non-reciprocal homoeologous rearrangements (a) at each generation for all four resynthesized Brassica napus populations (lines connect data point from the same lineage). Per population, we also compared (b) the number of homoeologous rearrangements and (c) the average size of these rearrangements across all individuals. Boxplots represent interquartile range, horizontal line, the median with minimum and maximum values in the data. Letters above represent significativity after $t$-test $(p<0.01)$.

Figure 4. Cumulative size of fixed non-reciprocal homoeologous rearrangements in $A$ and $C$ subgenomes in all individuals at each generation for all four resynthesized Brassica napus populations. Letters in each graph represent the overall significant differences between populations ( $t$-test, $p<0.05$ ) whereas the stars represent significant differences in cumulative size between subgenomes in a generation (ttest, $\left.{ }^{*} \mathrm{p}<0.05, * * \mathrm{p}<0.01\right)$. 


\section{TABLE}

2 Table 1. Summary of homoeologous deletions having a significant impact on percentage of cells with univalents/multivalents and on the number of seeds per 3100 flowers in resynthesized Brassica napus individuals (see the Statistical analyses section in the Materials and Methods).

\begin{tabular}{|c|c|c|c|c|c|c|c|c|c|c|c|c|c|c|c|c|}
\hline & \multirow[b]{2}{*}{ 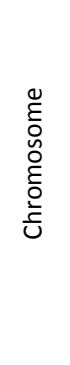 } & \multirow[b]{2}{*}{ 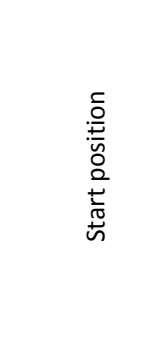 } & \multirow[b]{2}{*}{ 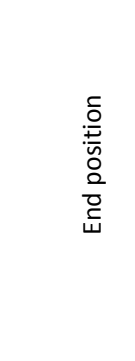 } & \multirow{2}{*}{ 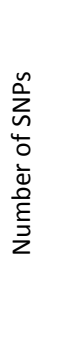 } & \multirow{2}{*}{ 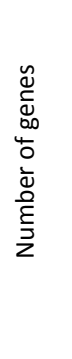 } & \multirow{2}{*}{ 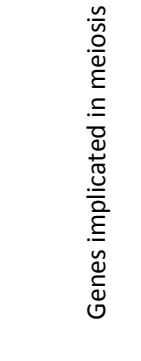 } & \multicolumn{2}{|c|}{ 'EMZ' individuals } & \multicolumn{2}{|c|}{ 'UG EMZ' individuals } & \multicolumn{2}{|c|}{ 'RCC' individuals } & \multicolumn{2}{|c|}{ 'CRC' individuals } & \multicolumn{2}{|c|}{ All individuals } \\
\hline $\begin{array}{c}\text { Phenotypic } \\
\text { trait }\end{array}$ & & & & & & & 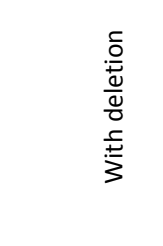 & 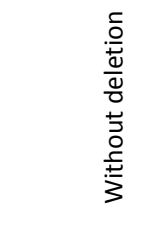 & 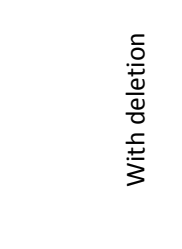 & 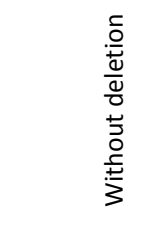 & 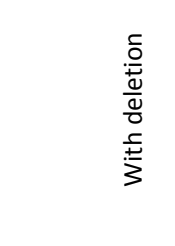 & 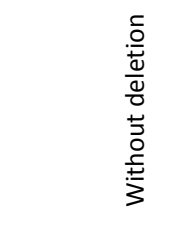 & 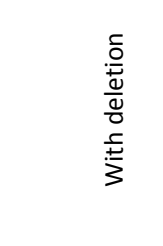 & 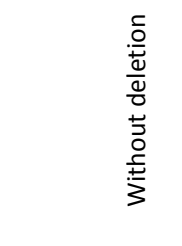 & 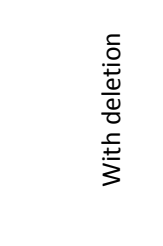 & 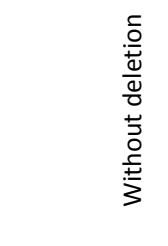 \\
\hline$\%$ of cells & & & & & & 1 (ML2, & 2 & 40 & & 58 & & & 2 & & $11\left(^{*}\right)$ & \\
\hline with & & & & & & AT2G4289 & $0.18 \%(0.6 \%$ & $0.26 \%(0.11$ & 3 & $0.26 \%(0.14$ & 4 & 27 & $0.24 \%(0.06$ & 32 & $0.19 \%(0.14$ & 157 \\
\hline multivalent & $\mathrm{A} 03$ & $7,593,332$ & $9,793,914$ & 16 & 440 & 0) & ) & $\%)$ & $0.12 \%(0.16 \%)$ & $\%)$ & $0.22 \%(0.16 \%)$ & $0.29 \%(0.09 \%)$ & $\%)$ & $0.30 \%(0.09 \%)$ & \%) & $0.27 \%(0.11 \%)$ \\
\hline$\%$ of cells & & & & & & & $5(*)$ & 36 & & 50 & & & $8(*)$ & & 20 & \\
\hline with 19 & & $17,970,61$ & $20,069,82$ & & & & $0.23 \%(0.14$ & $0.51 \%(0.14$ & 6 & $0.40 \%(0.16$ & 1 & 30 & $0.51 \%(0.08$ & 26 & $0.40 \%(0.15$ & 142 \\
\hline bivalents & $\mathrm{A} 02$ & 4 & 4 & 23 & 258 & 0 & $\%)$ & $\%)$ & $0.38 \%(0.10 \%)$ & $\%)$ & $0.50 \%$ & $0.50 \%(0.09 \%)$ & $\%)$ & $0.37 \%(0.16 \%)$ & $\%)$ & $0.44 \%(0.16 \%)$ \\
\hline & & & & & & 1 & & & & & & & & & & \\
\hline Number of & & & & & & (CDC20,1, & $5(*)$ & 40 & & 52 & 4 & 30 & $8\left(^{*}\right)$ & 29 & 27 & 151 \\
\hline seeds $/ 100$ & & & & & & AT4G3327 & $53.69(57.54$ & $90.73(62.16$ & $10(*)$ & $60.90(76.16$ & $108.53(117.0$ & $266.00(268.6$ & $60.68(69.70$ & $161.26(135.9$ & $55.70(63.42$ & $135.44(168.9$ \\
\hline flowers & $\mathrm{C} 01$ & $2,475,667$ & $8,275,217$ & 21 & 820 & 0) & ) & ) & 44.60(49.66) & ) & 2) & 1) & ) & 3) & ) & 4) \\
\hline Number of & & & & & & & 7 & 38 & $12\left(^{*}\right)$ & 50 & & 26 & & 32 & 32 & 146 \\
\hline seeds/100 & & & & & & & $97.26(68.02$ & $80.31(61.76$ & $111.57(108.5$ & $47.06(57.23$ & $8(*)$ & $269.17(261.7$ & $5(*)$ & $149.62(131.6$ & $82.51(82.77$ & $121.43(160.7$ \\
\hline flowers & $\mathrm{C} 02$ & $4,887,388$ & $6,416,485$ & 17 & 233 & 0 & ) & 1 & 1) & 1 & $23.24(5.53)$ & 5) & $24.99(3.12)$ & 5) & 1 & 1) \\
\hline Number of & & $44,901,24$ & $46,386,41$ & & & & $9\left(^{*}\right)$ & 36 & $\left.122^{*}\right)$ & 50 & 2 & 32 & $7\left(^{*}\right)$ & 30 & 30 & 148 \\
\hline seeds $/ 100$ & $\mathrm{CO} 4$ & 1 & 0 & 45 & 228 & 0 & $43.00(44.28$ & $92.04(62.82$ & $16.11(22.03)$ & $63.85(72.05$ & $181.01(162.3$ & $251.63(265.5$ & $62.33(59.33$ & $157.52(136.7$ & $47.13(65.05$ & $129.60(161.8$ \\
\hline
\end{tabular}




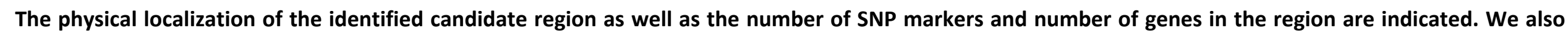

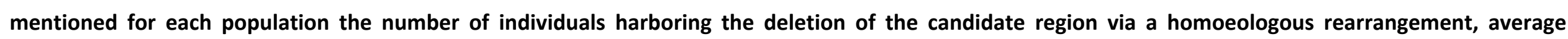

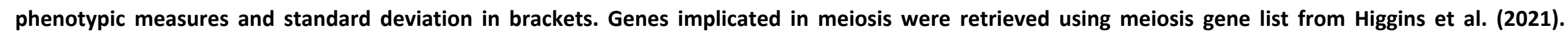

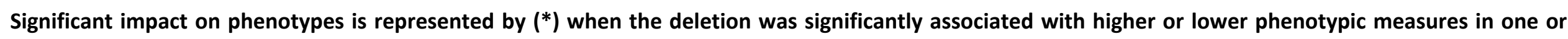
several genetic backgrounds. 
B. oleracea ' $R C$ ' CC, $2 \mathrm{n}=2 \mathrm{x}=18$

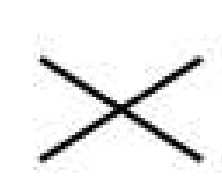

B. rapa 'C1.3' AA, $2 n=2 x=20$ or $B$. rapa 'C1.3' $A A, 2 n=2 x=20$

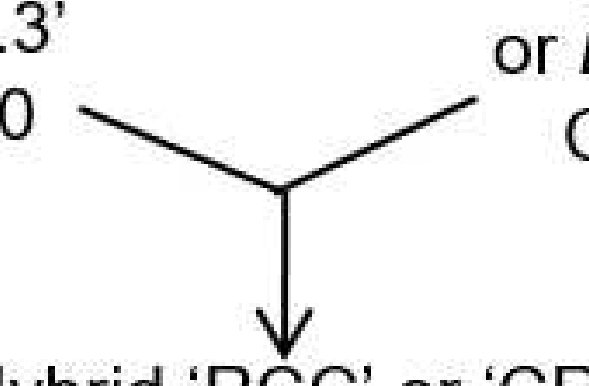
or B. oleracea 'RC' CC, $2 n=2 x=18$

F1 Hybrid 'RCC' or 'CRC' AC, $2 n=2 x=19$

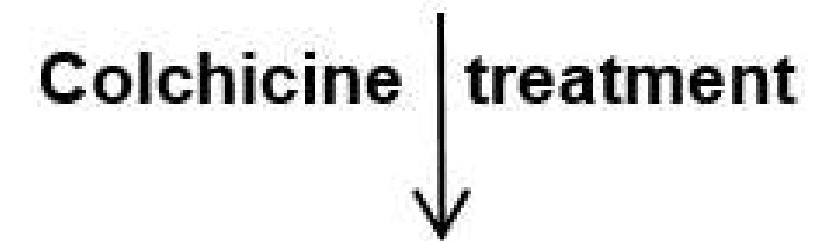

'RCC-SO' or 'CRC-SO' resynthesized $B$. napus AACC, $2 n=4 x=38$

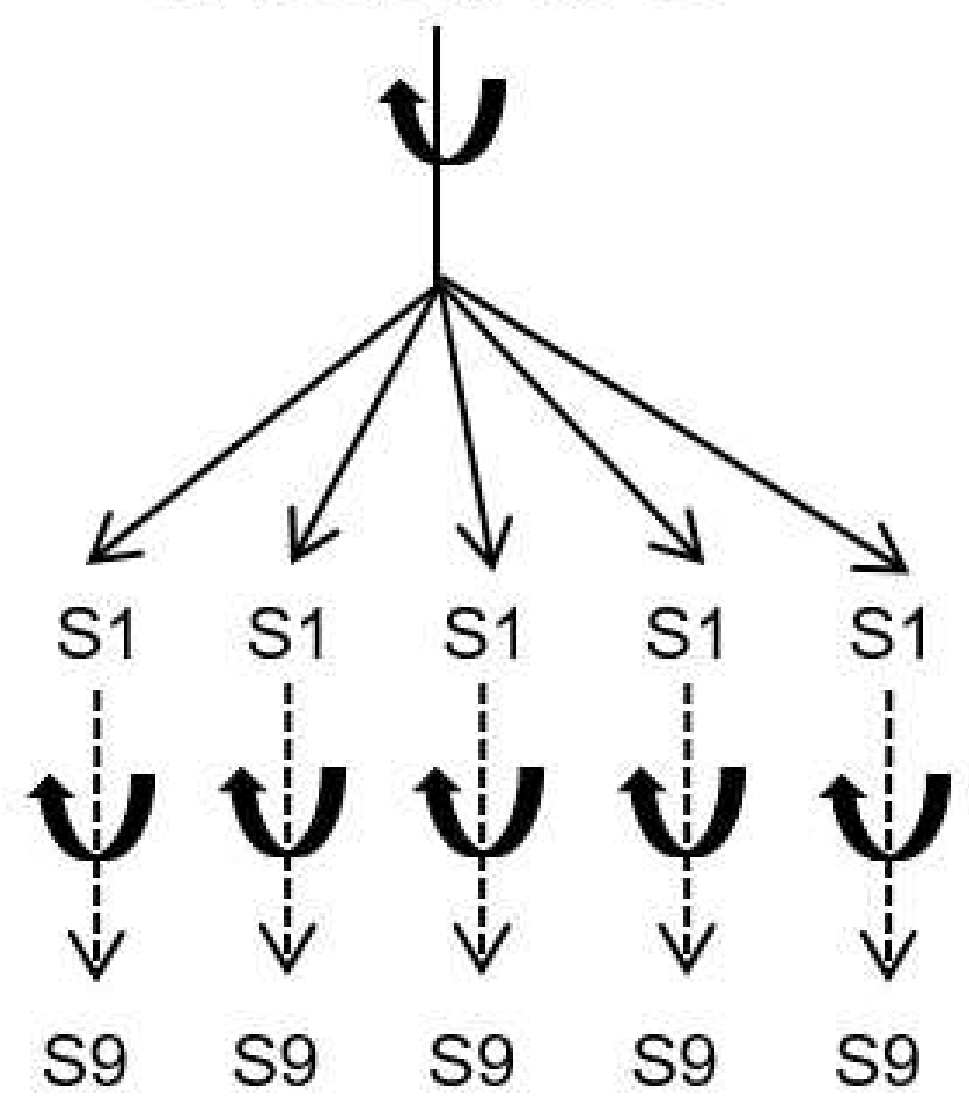

B. oleracea 'HDEM' CC, $2 n=2 x=18$<smiles>C1CC2(C1)CC2</smiles>

B. rapa ' $\mathrm{Z1}$ '

AA, $2 n=2 x=20$

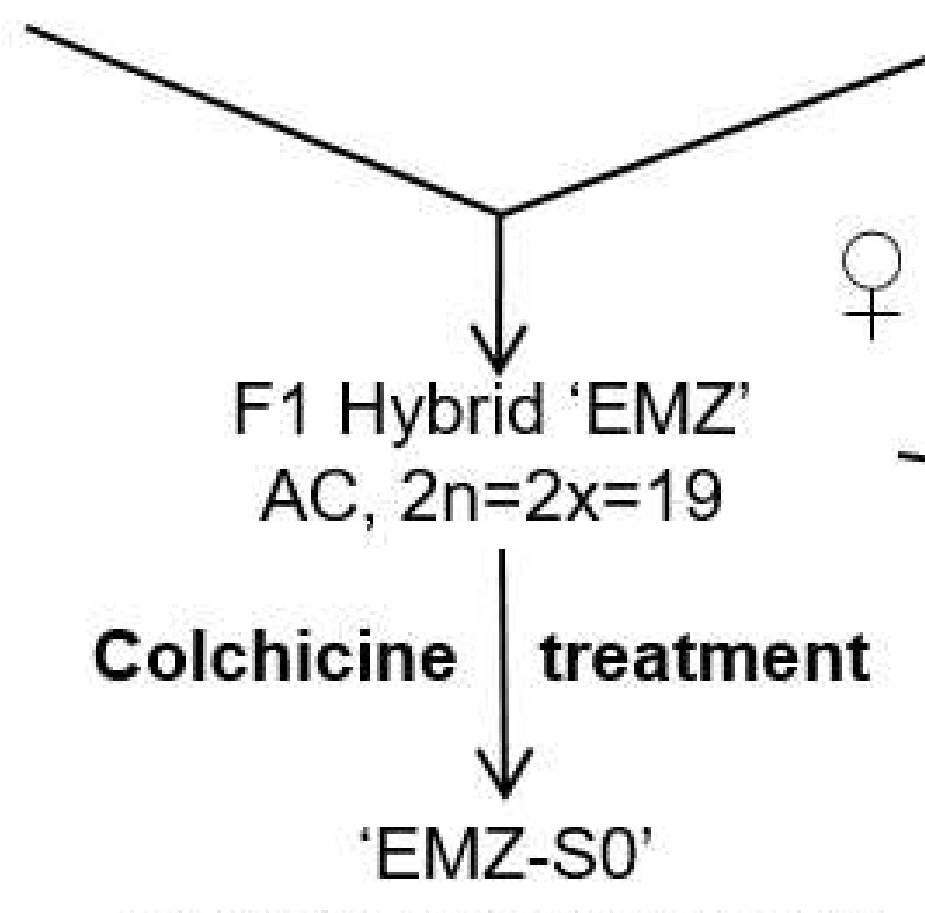

resynthesized $B$. napus AACC, $2 n=4 x=38$
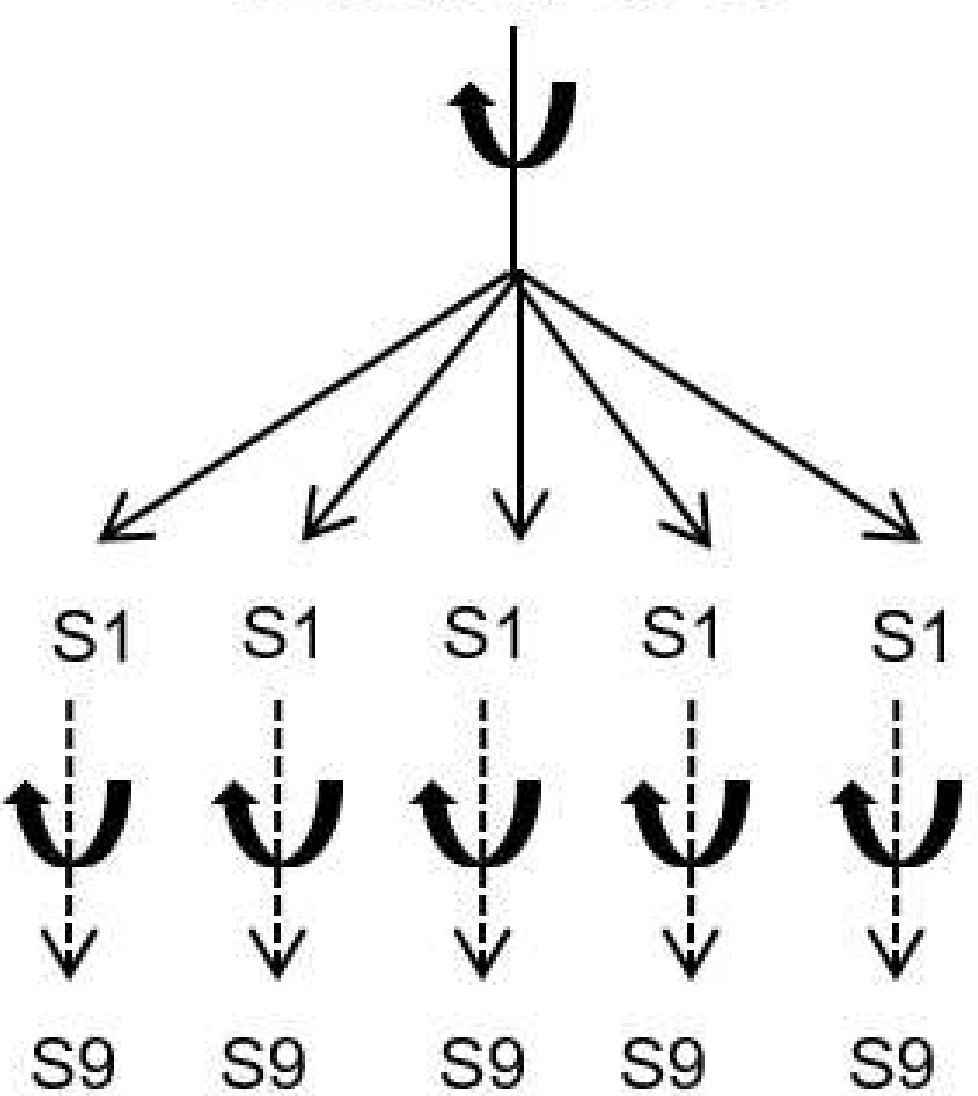

'EMZ-SO'

resynthesized $B$. napus AACC, $2 n=4 x=38$

Unreduced gametes

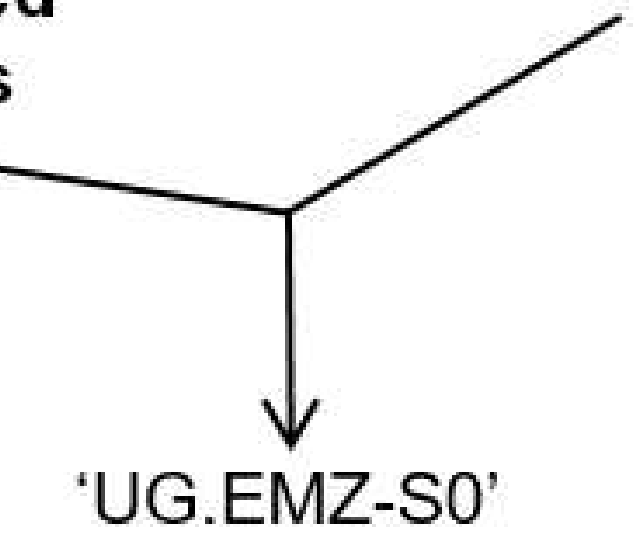

resynthesized $B$. napus AACC, $2 n=4 x=38$
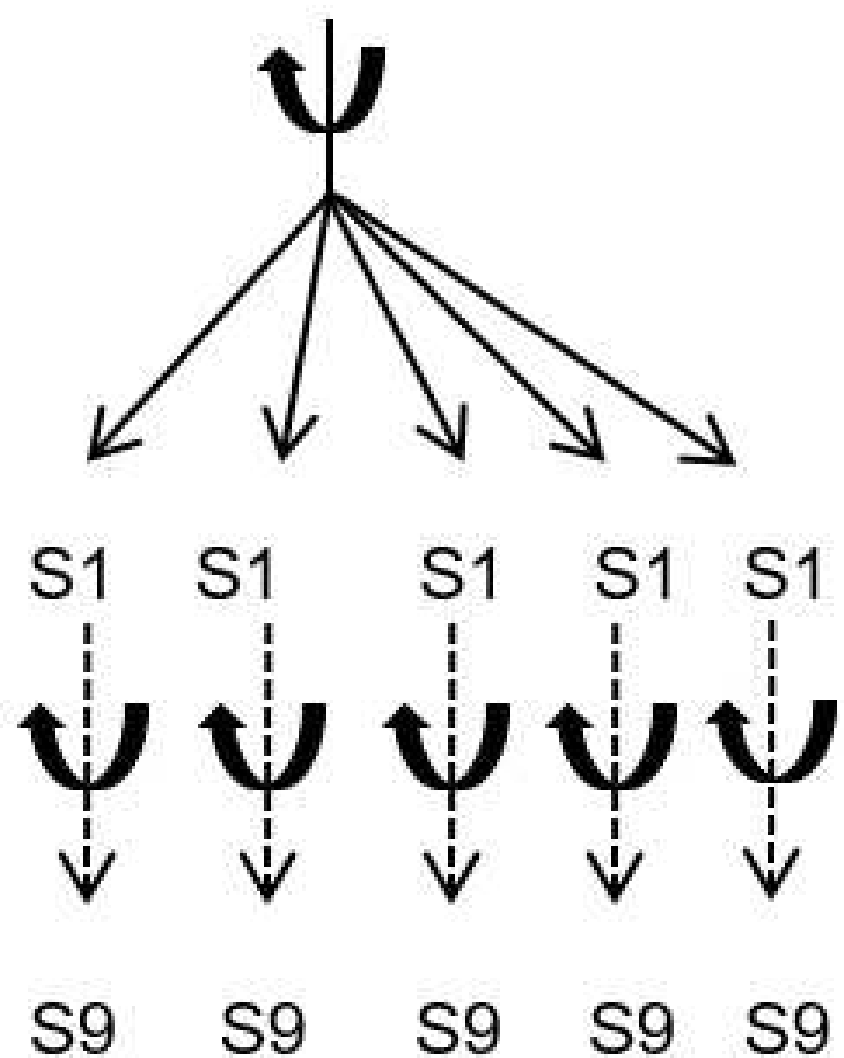

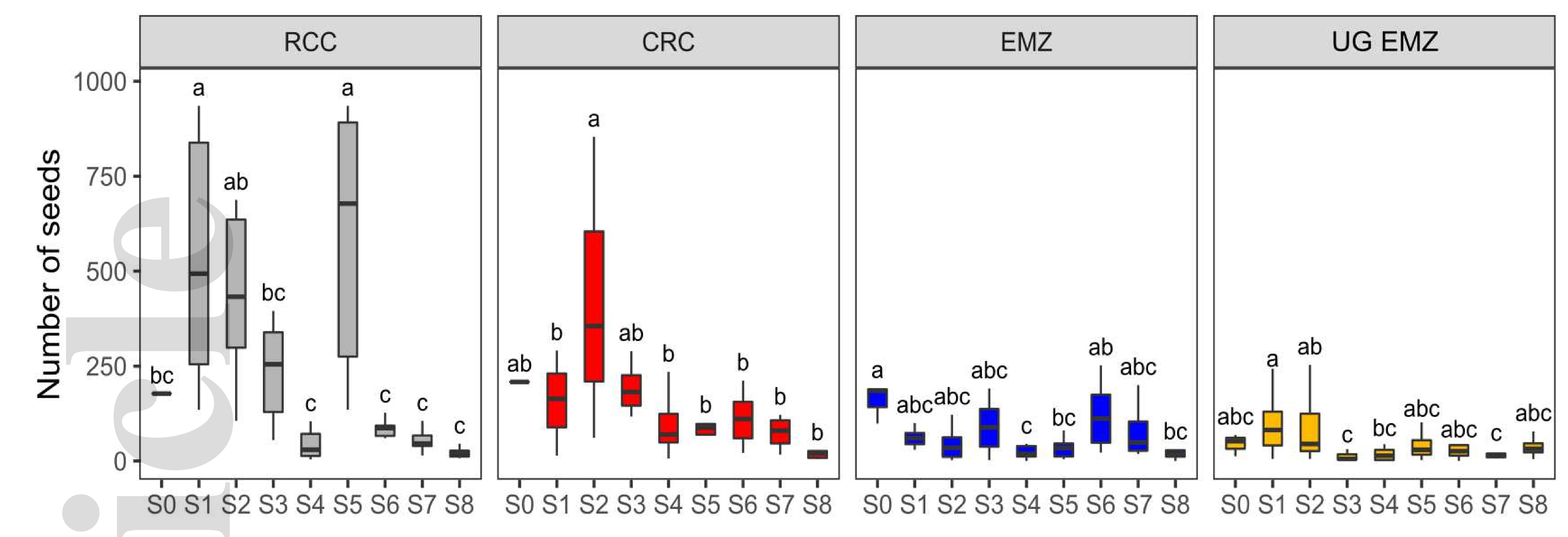

Crosses
RCC
CRC
ENZ
帛 UGEMZ
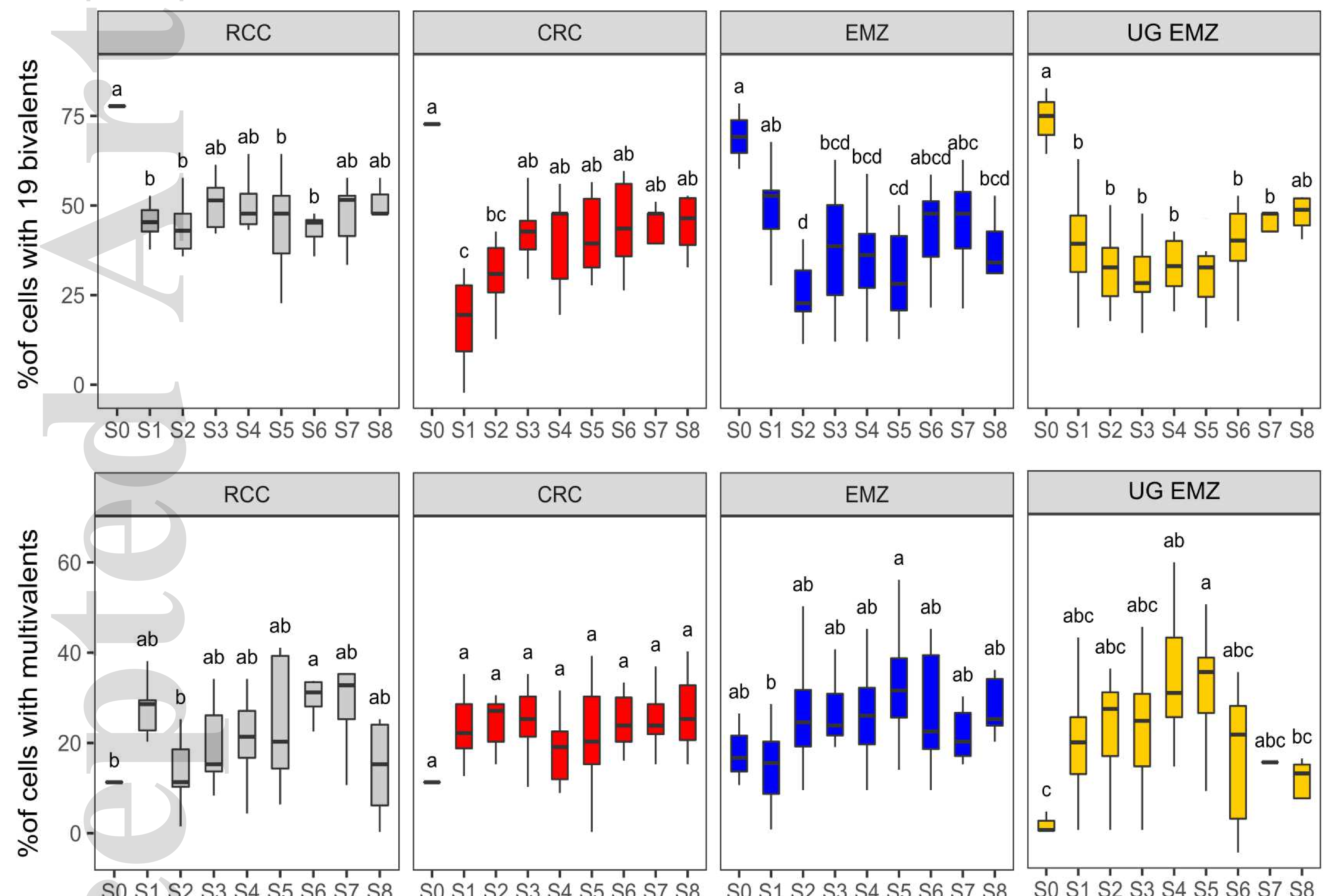

Generations 
